\title{
Electron transport in a nanowire irradiated by an intense laser pulse
}

\author{
J. F. Ong $\odot,{ }^{*}$ P. Ghenuche $\odot,{ }^{\dagger}$ and K. A. Tanaka $\odot^{*}$ \\ Extreme Light Infrastructure-Nuclear Physics (ELI-NP), "Horia Hulubei” National Institute for Physics and Nuclear Engineering \\ (IFIN-HH), 30 Reactorului Street, RO-077125 Bucharest-Măgurele, Romania
}

(Received 25 February 2021; accepted 31 August 2021; published 17 September 2021)

\begin{abstract}
Electron transport in a nanowire exhibits a distinct behavior following the irradiation of intense laser pulse. Using particle-in-cell simulation, we observe a large-amplitude particle-driven wakefield excitation followed by electron acceleration in the solid density. Besides, we observed the quiver of the electrons across the nanowire under the action of the surrounding laser electric field facilitating deeper wakefield propagation in the nanowire with $2.5 \times$ energy gain over a flat target. These results open insights into the laser-energy coupling with nanostructure targets and radiation sources, and motivate the wakefield acceleration in solid density plasma.
\end{abstract}

DOI: 10.1103/PhysRevResearch.3.033262

\section{INTRODUCTION}

The interaction of high-power lasers with nanostructure targets received a lot of attention lately due to their increased light-matter coupling efficiency [1-4]. A laser pulse of relativistic intensity can heat a nanowire target to a much larger depth over a flat solid target [5]. Such an efficient laser energy absorption opens up various applications such as enhanced ion acceleration [6], attosecond bunch generation [7,8], enhanced x-ray [9-13], and gamma-ray generation [14], as well as efficient microfusion $[5,15,16]$. Therefore, understanding the electron dynamics and transport in the nanostructure is fundamental for the control of these applications.

Numerous studies were carried out to investigate the electron transport in the nanowires. The fast electrons generated upon laser irradiation are collimated in the nanowire arrays for a long time by the resistive magnetic field, typically in the time frame of picosecond as reported in Ref. [17]. The laser energy absorption was mainly attributed to the vacuum heating through the laser electric field normal to the nanowire surfaces $[18,19]$ and was utilized to optimize the electron acceleration [20].

Typically, the electron acceleration and transport in the nanowire occur in different stages. The electric field of the laser tears off electrons from the surface of the nanowire and accelerates them in the forward direction by the magnetic field, forming electron bunches outside the nanowire. These bunches introduce a return current at the skin layer to fulfill the requirement of local current neutrality [21]. The return

\footnotetext{
*jianfuh.ong@eli-np.ro

†petru.ghenuche@eli-np.ro

*kazuo.tanaka@eli-np.ro
}

Published by the American Physical Society under the terms of the Creative Commons Attribution 4.0 International license. Further distribution of this work must maintain attribution to the author(s) and the published article's title, journal citation, and DOI. current generates a quasistatic azimuthal magnetic field that pinches the nanowire radially inward. This nanoscale $Z$ pinch is capable of forming an ultradense electron bunch, surpassing 1000 times the critical density [15]. The laser continues to propagate in the space between the wires and heats the plasma until the nanowire blowout fills the space completely. Until now, the electron dynamics in the nanowire which contributed to the high laser energy absorption have not been addressed in detail and demand further clarification. Since real-time observation of electron transport across the nanowire is experimentally difficult, it is of critical importance to understand electron transport by using numerical simulation with a high spatial and temporal resolution to identify the absorption dynamics.

In this paper, the electron dynamics accounted for the enhanced laser energy absorption of a nanowire irradiated by intense laser pulse are reported. Particle-in-cell (PIC) simulations show that a wakefield is excited by the $2 \omega_{\mathrm{L}}$ fast electron bunches generated by the $\mathbf{J} \times \mathbf{B}$ mechanism at the tip of the nanowire. This wakefield has an amplitude of the order of $\mathrm{TV} \mathrm{m}{ }^{-1}$, oscillating at the plasma frequency and propagates deep into the nanowire. Electrons injected at the later stage are accelerated by the wakefield when the right initial conditions are satisfied. The laser fields then brush up the side surfaces and swing the electron across the nanowire. These electrons assist the wakefield to propagate deeper into the nanowire with $2.5 \times$ energy gain over a flat target.

\section{METHODS}

The simulations were performed using two-dimensional (2D) PIC code EPOCH [22]. The simulation box is discretized by $4069 \times 256$ cells with the spatial resolution $\Delta x=$ $1.97 \mathrm{~nm}, \Delta y=7.81 \mathrm{~nm}$, and a temporal resolution $\Delta t=$ 3.16 as. The grid sizes are small enough to resolve the plasma wavelength $\lambda_{\mathrm{p}}$ at $1000 n_{\mathrm{cr}}$, which is $\sim 25 \mathrm{~nm}$. Here, $n_{\mathrm{cr}}$ is the critical density. Each cell consists of ten macroparticle ion species. Field ionization and mobile ion are implemented, but collisional ionization is not included. The fifth order 
(a)
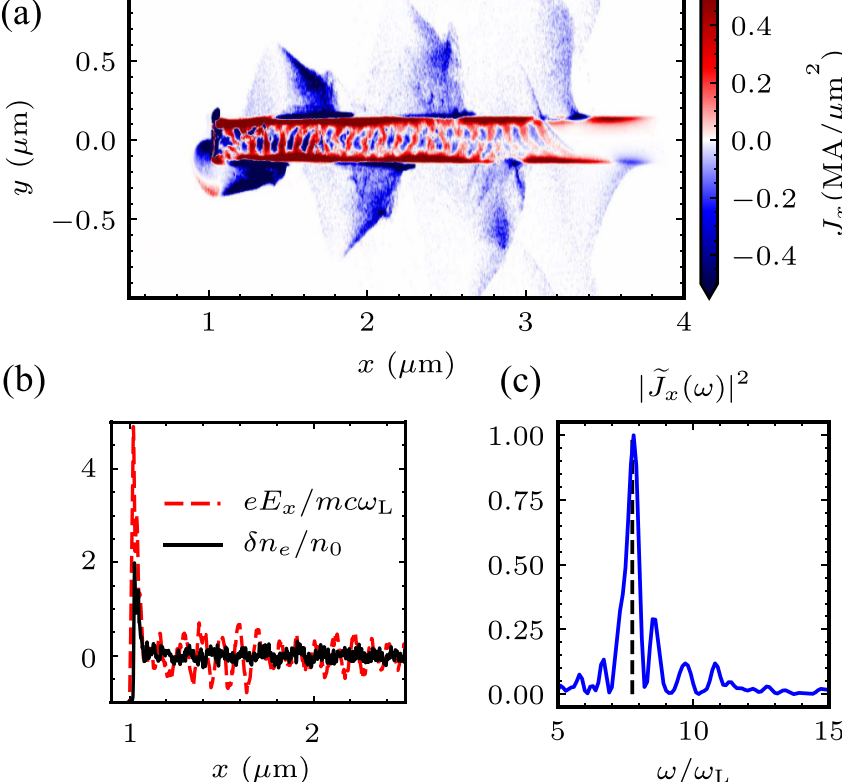

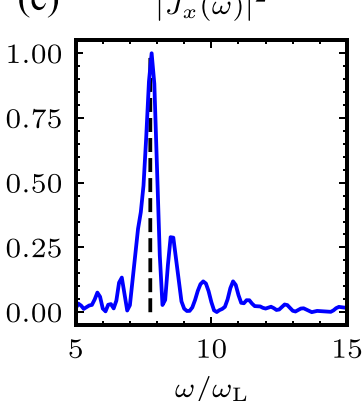

FIG. 1. (a) Snapshot of the current density $J_{x}$ at $t=13 \mathrm{fs}$. The laser pulse travels from left to right. Electrons are stripped off from the surface of the nanowire and moved to the right, indicated by the forward current (blue). The return current (red) is created at the surface of the nanowire. A plasma wave is excited inside the nanowire as indicated by the sinusoidal current density. (b) The longitudinal density perturbation $\delta n_{e} / n_{0}$ (solid line) and wakefield $E_{x}$ (dashed line in normalized unit) at $y=0$. Here, $n_{0}=60 n_{\mathrm{cr}}$ is the average electron density created by the field ionization along the nanowire. (c) The frequency spectrum associated with the current density shown in (a). The dashed line indicates the plasma frequency of $\omega_{\mathrm{pe}}=7.75 \omega_{\mathrm{L}}$.

particle shape function and the current smoothing are used to suppress the numerical heating. The laser pulse is modeled as a linearly polarized plane wave with the central wavelength $\lambda_{\mathrm{L}}=0.8 \mu \mathrm{m}$ and a peak intensity $I_{0}=10^{22} \mathrm{~W} \mathrm{~cm}^{-2}$ $\left(a_{0}=68\right)$, simulating the conditions available at ELI-NP [23]. The laser is irradiated onto the nanowire at normal incidence. Here, $a_{0}=e E_{0} /\left(m c \omega_{\mathrm{L}}\right)$ is the normalized laser amplitude, where $E_{0}$ is the peak laser field strength, $\omega_{\mathrm{L}}=2 \pi / \lambda_{\mathrm{L}}$ is laser central frequency, and $e$ and $m$ are the charge and mass of electron. The laser temporal profile is $a(t)=a_{0} \exp \left[-\left(t-t_{0}\right)^{2} /\right.$ $\tau_{L}^{2}$ ] with $\tau_{L}=13.2 \mathrm{fs}$ (22 fs full width at half maximum), and $t_{0}=2 \tau_{L}$. A carbon nanowire with length $L=5 \mu \mathrm{m}$ and diameter $d=300 \mathrm{~nm}$ is placed horizontally at the center of the simulation box. The carbon nanowire has a steplike profile with density $0.52 \mathrm{~g} \mathrm{~cm}^{-3}\left(n_{i}=15 n_{\mathrm{cr}}\right)$. The initial charge state of the carbon atoms is zero.

\section{RESULTS AND DISCUSSIONS}

\section{A. 2D PIC simulation results}

The current density $J_{x}=-e n_{e} v_{x}$ at $t=13 \mathrm{fs}$ after laser irradiation is illustrated in Fig. 1(a). The laser first ionizes the carbon atoms at the surface of the nanowire. Then, the $E_{y}$ component of the laser field strips off electrons and accelerates them forward by the $B_{z}$ component. A return current is then generated on the surface of the nanowire to main- tain the current quasineutrality, where the forward current is negative (blue) and the return current is positive (red). In addition, the electron displacement from the plane of the nanowire tip $(x \leqslant 1 \mu \mathrm{m})$ is observed. This is caused by the $\mathbf{J} \times \mathbf{B}$ heating [24] due to the oscillating term of the ponderomotive force of the linearly polarized laser field incident normally on the target. Details of the acceleration and electron bunching mechanism outside the nanowire have been discussed in Refs. [7,8]. Hereafter, we focus on the electron behavior inside the nanowire. A sinusoidal current inside the nanowire is generated soon after laser irradiation [see Fig. 1(a) and Supplemental Material (SM) Video 1 [25]]. This current has an amplitude of around $\left|j_{x}\right|=0.4 \mathrm{MA} / \mu \mathrm{m}^{2}$, which corresponds to a current of $28 \mathrm{kA}$ considering the area of the nanowire with diameter $d$. The electron velocity derived from this current density has a value of $v_{x} \sim 0.08 c$. The electric field component $E_{x}$ and the density perturbation $\delta n_{e} / n_{0}=n_{e} / n_{0}-1$ are shown in Fig. 1(b). The constant $n_{0}=60 n_{\text {cr }}$ is the average electron density created by field ionization along the nanowire. However, the maximum density at the plane of the nanowire tip is $n_{e}=3 n_{0}=180 n_{\mathrm{cr}}$ due to the compression caused by the laser radiation pressure. The relativistic effects of the present laser intensity increase the cutoff density for the laser penetration up to $n_{\mathrm{cr}}^{\prime}=48 n_{\mathrm{cr}}\left(n_{\mathrm{cr}}^{\prime}=\gamma n_{\mathrm{cr}}, \gamma=\sqrt{1+a_{0}^{2} / 2}\right)$. The nanowire is opaque to the laser field even under self-induced transparency condition, and hence rules out the penetration of the laser field into the nanowire. In conjunction with the density, $E_{x}$ has a peak of approximately $2.4 \mathrm{TV} \mathrm{m}^{-1}$, which corresponds to $e E_{x} /\left(m c \omega_{\mathrm{L}}\right) \approx 0.6$. This nanoscale charge separation field is $10 \times$ larger in magnitude and a thousand times smaller in scale than the one that can be achieved by a typical laser wakefield acceleration in an underdense plasma. Further analysis of the current density spectrum gives a spike at plasma frequency $\omega_{\mathrm{pe}}=\omega_{\mathrm{L}} \sqrt{n_{0} / n_{\mathrm{cr}}}=7.75 \omega_{\mathrm{L}}$ as shown in Fig. 1(c). Together with the large amplitude $E_{x}$, these signs point towards the wakefield excitation by a driving force inside the nanowire.

The driving force for the wakefield excitation is attributed to the electron bunches generated by the $\mathbf{J} \times \mathbf{B}$ heating at the nanowire tip. Typically, electron bunches generated in this way are separated by $0.5 \lambda_{\mathrm{L}}$ interval or at $2 \omega_{\mathrm{L}}$ frequency as evident in Fig. 2. They are accelerated into the nanowire with large forward momentum $p_{x} /(m c) \sim 20$. Due to the small diameter of the nanowire, these bunches are not clearly visible from the current density. The longitudinal momentum of the bulk electrons shown in the inset of Fig. 2 exhibits a wavelike structure with the amplitude $\approx 0.08$, consistent with the electron velocity derived earlier. The peak-to-peak interval is the plasma wavelength $\lambda_{\mathrm{p}}=2 \pi v_{\mathrm{p}} / \omega_{\mathrm{p}} \approx 100 \mathrm{~nm}$, where $v_{\mathrm{p}}=0.97 \mathrm{c}$ is the plasma wave phase velocity. Note that the phase space in Fig. 2 is restricted to $-0.1 \mu \mathrm{m} \leqslant y \leqslant 0.1 \mu \mathrm{m}$, such that electrons in the transverse skin layer and those outside the nanowire are excluded.

To understand better the electron transport in the nanowire, we present the electron macroparticles for the energy $E /\left(m c^{2}\right)=\gamma \geqslant 2$ and the $E_{y}$ component of the electric field in Fig. 3(a). The $2 \omega_{\mathrm{L}}$ electron bunches can now be readily observed inside the nanowire. We noticed that part of the electrons stripped off by the laser $E_{y}$ component during the 


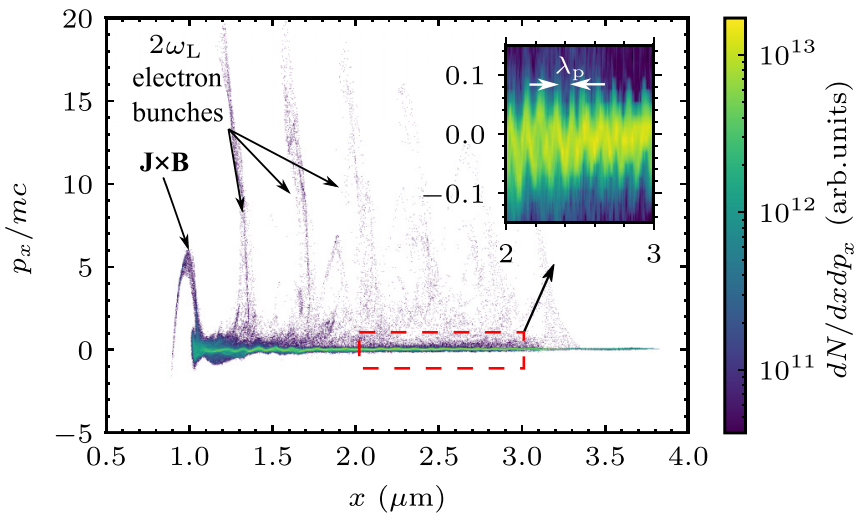

FIG. 2. Longitudinal electron phase space at $t=13 \mathrm{fs}$, demonstrating the presence of $2 \omega_{\mathrm{L}}$ electron bunches inside the nanowire. The phase space is restricted at $-0.1 \mu \mathrm{m} \leqslant y \leqslant 0.1 \mu \mathrm{m}$ to exclude electrons in the skin layer and outside the nanowire. The inset shows the enlarged phase space for $2 \mu \mathrm{m} \leqslant x \leqslant 3 \mu \mathrm{m}$ with the plasma wavelength $\lambda_{\mathrm{p}} \approx 100 \mathrm{~nm}$.

first half cycle are pushed back to the nanowire at the second half cycle, similar to the vacuum heating mechanism $[18,19,26]$. A considerable amount of electrons right behind each main bunch are returning to the nanowire [see the SM for Video 1 and Fig. S1(a) [25]]. Vacuum heating is an efficient absorption mechanism when the electron oscillation amplitude is larger than the preplasma length. However, a detailed analysis of the electron trajectories shown in Fig. 3(a)

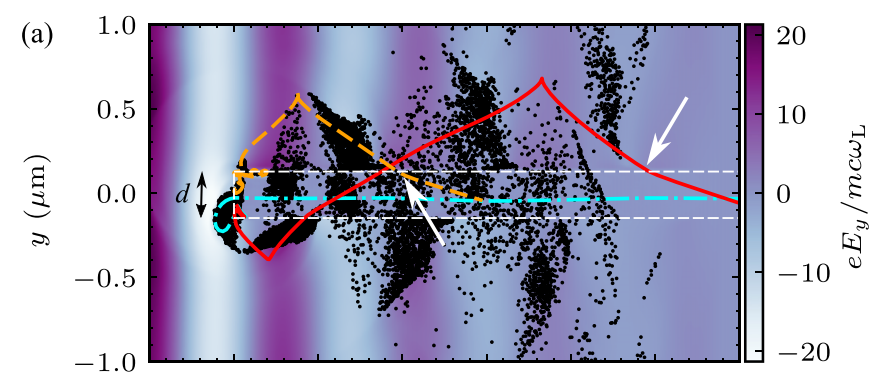

(b)

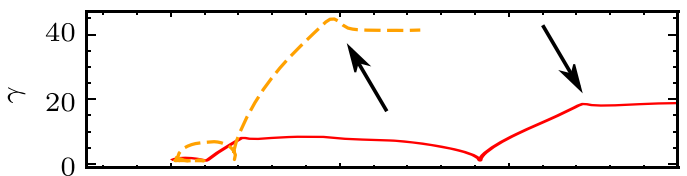

(c)

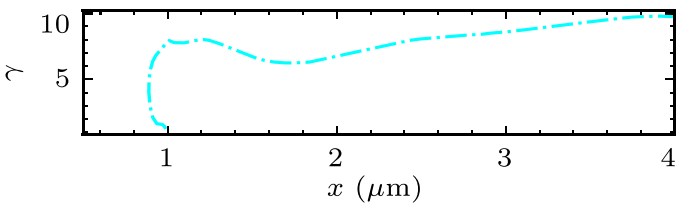

FIG. 3. (a) Snapshot of electron macroparticles with $\gamma \geqslant 2$ at $t=13 \mathrm{fs}$. Electrons outside the nanowire move in the $+y$ direction when $E_{y}<0$ and in the $-y$ direction when $E_{y}>0$, respectively. The $2 \omega_{\mathrm{L}}$ electron bunches inside the nanowire propagates in the forward direction. The solid, dashed, and dash-dotted lines indicate three trajectories of three sample electrons until $t=20 \mathrm{fs}$. (b),(c) The $\gamma$ of the corresponding electrons in (a). The arrows in (a) indicate the electrons entering the nanowire boundary and their corresponding $\gamma$ in (b). The dashed white line indicates the boundary of the nanowire of diameter $d$.

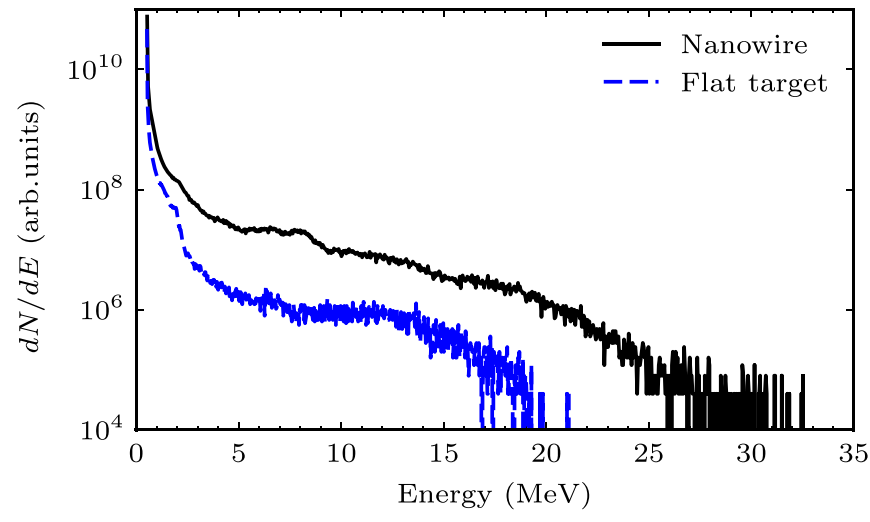

FIG. 4. Electron energy spectra at $t=20 \mathrm{fs}$ for nanowire (solid line) and flat target (dashed line) within the region $-0.1 \mu \mathrm{m}$ $\leqslant y \leqslant 0.1 \mu \mathrm{m}$.

(solid and dashed lines) reveals that the electron is swinging across the nanowire with an oscillating amplitude much larger than the nanowire dimension itself. The electron oscillation amplitude is now $y_{\mathrm{osc}}=a_{0} c / \omega_{\mathrm{L}}>d$, pointing to a laser energy absorption mechanism different from vacuum heating. This is nothing but a relativistic electron motion in an electromagnetic plane wave with no net energy gain unless several assumptions are violated, for instance, limiting the interaction region and introducing static electric or magnetic fields as discussed in Ref. [26]. In the present case, there is a wakefield $E_{x}$ inside the nanowire [see Fig. 2(b)], and a quasistatic magnetic field at the boundary induced by the return current. The entering electrons (i.e., supplied by the laser electric field, $E_{y}$ ) pointed by the arrows in Fig. 3(a) are mostly located at $E_{x} \approx 0$, leading to the motion with constant $\gamma$ as evidenced in Fig. 3(b). Some electrons can surf with the wake wave but do not have sufficient time to be accelerated before exiting the nanowire. Outside the nanowire, the electron gains and loses energy depending on its position in the laser phase, e.g., the energy is zero at its maximum amplitude. In general, the energy of the electron is locked only when it is inside the nanowire.

On the other hand, the electron generated by the $\mathbf{J} \times \mathbf{B}$ heating at the tip is continuously injected into the nanowire. These electrons are shown by the dash-dotted line in Fig. 3(a), and their corresponding normalized energy is shown in Fig. 3(c). The energy of the electrons that enter the nanowire is $\gamma \sim 8$ and then drops to $\gamma \sim 6$ after propagating for $1.8 \mu \mathrm{m}$. The energy then increases gradually to $\gamma \sim 10$. The wakefield is responsible for the electron acceleration as it is the only longitudinal electric field inside the nanowire.

To compare the electron energy gain inside the nanowire over a flat target, the electron energy spectra are taken at $t=20 \mathrm{fs}$ are shown in Fig. 4. Beyond this point, the nanoscale $Z$ pinch begins and leads to the collapsing of the wakefield. In addition, the ion response time scale to the wakefield, $2 \pi / \omega_{\mathrm{pi}}$ at $n_{0}$ is also about $\sim 20 \mathrm{fs}$. Here, $\omega_{\text {pi }}$ is the ion plasma frequency. Therefore, the electron energy spectrum is recorded up to this point to minimize the effects of pinching and ion response. For a fair comparison, the energy spectra are restricted to $-0.1 \mu \mathrm{m} \leqslant y \leqslant 0.1 \mu \mathrm{m}$ in nanowire and flat target. The energy spectra show similar enhancement to the 


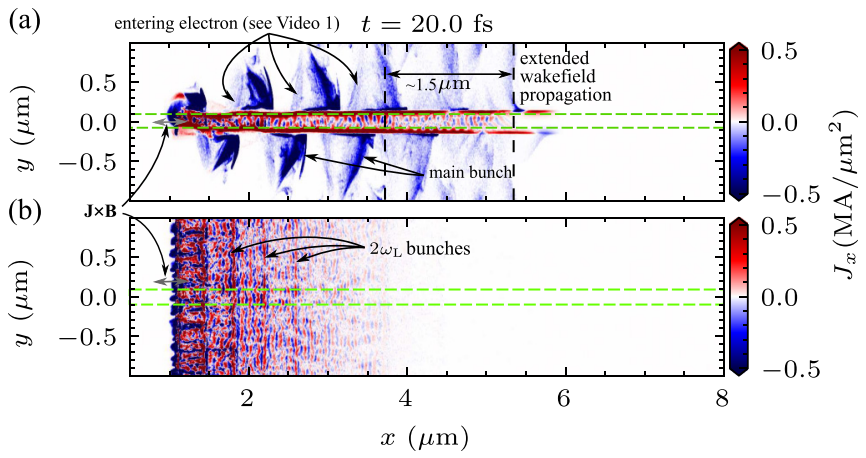

FIG. 5. The current density at $t=20 \mathrm{fs}$ for (a) nanowire, and (b) flat target. Both targets have the same length. The energy spectra are calculated for the region between the green lines. The arrows indicate the physical processes with the terms used in the text. measurements reported in Refs. [1,27]. The total electron energy inside the nanowire is evaluated to be 16 and $6.4 \mathrm{~mJ} / \mu \mathrm{m}$ for the flat target. Generally, the nanowire overcomes the flat target about $2.5 \times$ in electron energy gain. This is because the amplitude of the wakefield attenuates with distance into the target due to the velocity dispersion of the $2 \omega_{\mathrm{L}}$ bunches and the interaction with the wake induced within the bunch itself [28]. But in the nanowire, the energetic entering electrons act as a drive bunch, continuously exciting the wakefield during the nanowire crossing and lead to the extended propagation in the nanowire over a flat target by $1.5 \mu \mathrm{m}$ as shown in Fig. 5 . This subsequently results in enhanced energy absorption. In addition, the cutoff electron energy for the nanowire case is almost double the flat target. The collisional damping of the wakefield was reported in Ref. [29] as a possible source of heating, however, the inclusion of collisional effects (binary collision and collisional ionization) did not affect the results (see the SM for Fig. S2 [25]). The low $Z$ carbon (a)
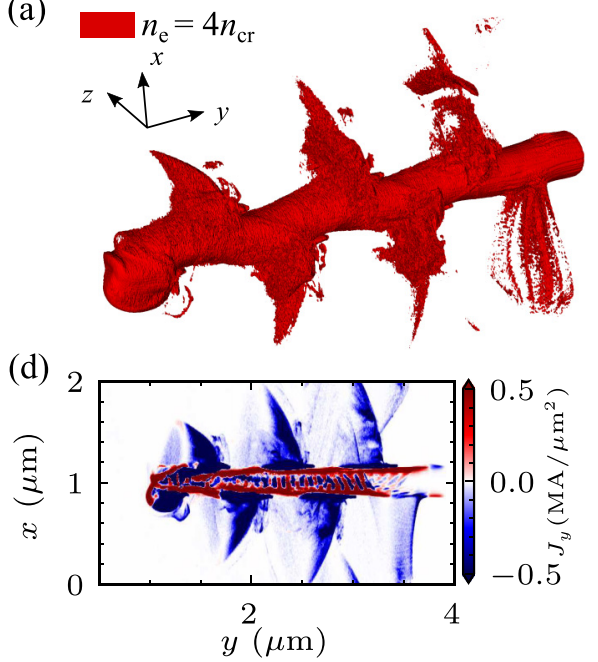

(b)

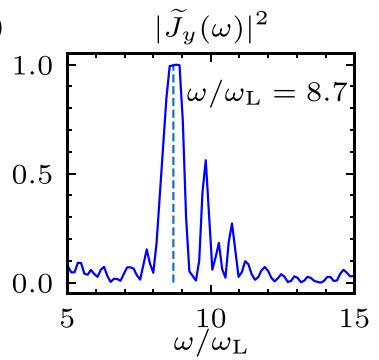

(e)

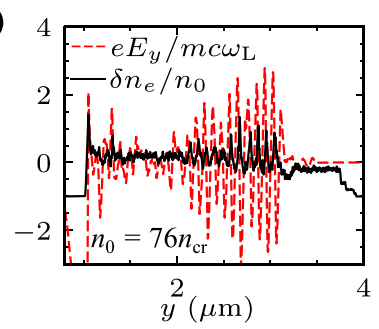

(c)

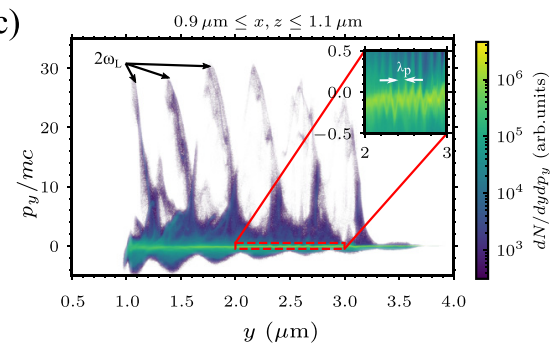

(f)

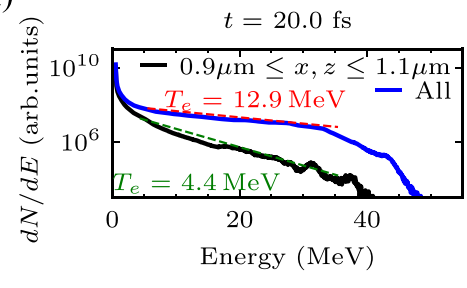

(g)

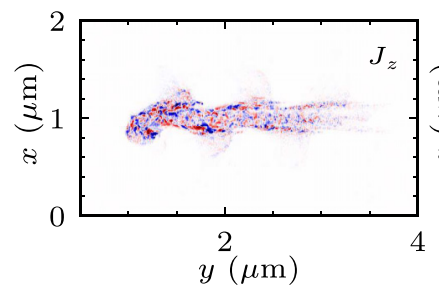

(h)

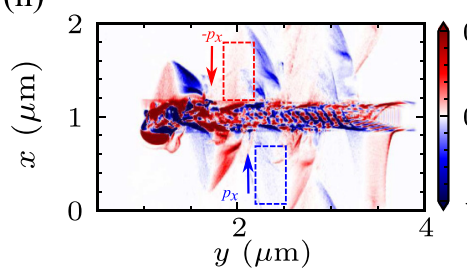

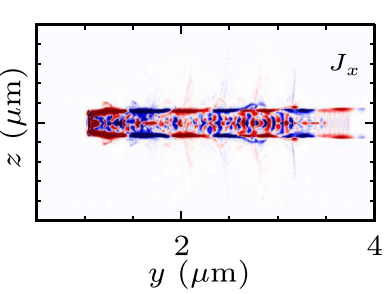

(i)

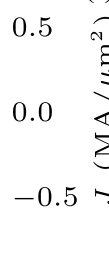

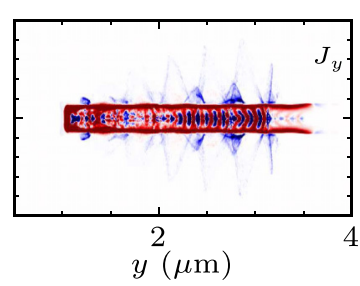

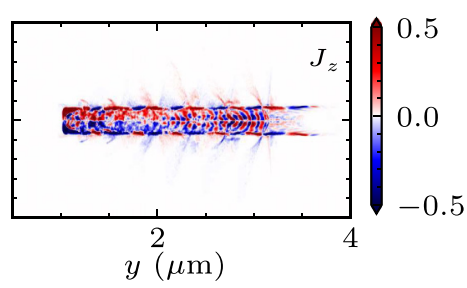

(j)

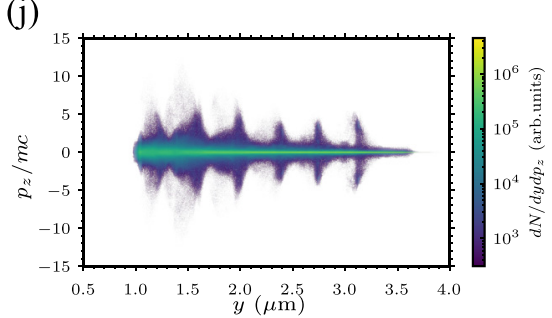

FIG. 6. 3D simulation results with PICONGPU code for linearly polarized laser field at $t=13 \mathrm{fs}$. (a) The isosurface of electron density at $n_{e}=4 n_{\mathrm{cr}}$. (b) The frequency spectrum associated with the longitudinal current density $J_{y}$. (c) The longitudinal electron phase space inside the nanowire. (d) The current density components, $J_{y}$ taken at $z=1 \mu \mathrm{m}$. (e) The longitudinal density perturbation (solid line) and wakefield (dashed line) along the nanowire center. (f) The total electron energy spectra at $t=20 \mathrm{fs}$ with the one inside the nanowire. (g) The transverse current components. (h) The transverse current density $J_{x}$ where the dashed boxes indicate the returning electron that will cross the nanowire with the corresponding transverse momentum, $p_{x} /(m c)$ in (i). (j) The transverse momentum component $p_{z} /(m c)$. 
plasma suggests that the radiation cooling time exceeds the hydrodynamic expansion by a few orders of magnitude up to the density $1 \times 10^{25} \mathrm{~cm}^{-3}$ with temperature in the range of $\mathrm{keV}-\mathrm{MeV}$ [15]. The energy dissipation through $\mathrm{x}$-ray emission and ion response can be assumed negligible.

\section{B. 3D PIC simulation results}

2D benchmark simulations were performed using PICONGPU code [30], which reproduce the primary results presented above. 3D simulations with both codes also support the observation of wakefield excitation. The $3 \mathrm{D}$ simulation results by using PICONGPU code are shown in Fig. 6. For linearly polarized laser field, the plasma wavelength, wakefield phase velocity, fast electron temperature, and the energy gain remain similar to 2D simulation. The longitudinal electric field, density perturbation, and electron energy cutoff are stronger. These are shown in Figs. 6(a)-6(f), where the one-to-one comparison to $2 \mathrm{D}$ simulation is apparent. As expected, electron crossing remains unaffected in the $3 \mathrm{D}$ geometry despite the presence of a large transverse surface current [see Figs. 6(g)]. This is evident through the transverse phase space diagram, $p_{x} /(m c)$ in Fig. 6(i), which match the beam position of the $J_{x}$ component in Fig. 6(h). A small amount of $p_{z} /(m c)$ component also present inside the nanowire as shown in Fig. 6(j). This differs from the nanocluster interaction, where electrons circulating around the surface rather than oscillating through the structure $[31,32]$. On the other hand, the energy absorption rate by electrons trapped in the wakefield at the initial irradiation phase is $\sim 0.1 \%$. Despite the small conversion rate, the energy enhancement and wakefield penetration inside the nanowire outperform the flat target. It is worth mentioning that for circularly polarized laser field, the electron crossing and $\mathbf{J} \times \mathbf{B}$ heating is ineffective and the wakefield excitation is strongly suppressed (see the SM for benchmark and 3D simulation results [25]).

To the best of our knowledge, these results provide clear evidence of particle-driven wakefield excitation in a solid density nanowire. Detailed investigation of the electron trajectories improves the understanding of electron transport in the nanowire. A key finding was that the entering electron supplied by the surrounding laser electric field assists a deeper wakefield propagation in the nanowire over the flat target. This has an important implication for deep energy penetration and suggests an alternative mechanism for laser heating which may be possible only for nanostructured materials. To optimize the electron acceleration in the nanostructure, conditions such as structure dimension and injection conditions demand investigation in detail. Making full use of the accelerating gradient of $\sim 1 \mathrm{TeVm}^{-1}$, electron energy gain up to $1 \mathrm{GeV}$ may be expected within the length of $1 \mathrm{~mm}$.

\section{CONCLUSIONS}

In summary, we have reported the wakefield generation in a solid density nanowire immediately following the interaction with an intense laser pulse. The driving force of the wakefield is identified to be the $2 \omega_{\mathrm{L}}$ electron bunches generated by the $\mathbf{J} \times \mathbf{B}$ heating at the tip of the nanowire. The wakefield is oscillating at the plasma frequency and its amplitude can reach 2.4 $\mathrm{TV} \mathrm{m}^{-1}$. Electrons are subsequently injected and accelerated by the wakefield deep into the nanowire when the right initial conditions are met. In addition, the laser electric field normal to the nanowire surface swings the electron across the nanowire. We have shown that the electron oscillation amplitude is larger than the nanowire itself and the vacuum heating is not effective. Instead, these electrons act as drive bunch and have facilitated a deeper wakefield propagation into the nanowire which results in $2.5 \times$ energy gain over a flat target. These interplay open insights into the laser volumetric heating of nanostructure targets. The enhanced electron energy gain will offer efficient $\mathrm{x}$-ray and $\gamma$-ray sources when the supporting substrate is substituted by the high- $Z$ materials [33]. The observation of such a large-amplitude wakefield excitation may motivate the development of plasma wakefield acceleration in solid density plasma [34].

\section{ACKNOWLEDGMENTS}

J.F.O. thanks L. G. Huang for the fruitful discussion. P.G. acknowledges the support of the Romanian Ministry of Education and Research through the PN 19060105 and ELIRO FLAP (ELI-RO-2017-27) projects. K.A.T. acknowledges intriguing comments from A. Pukhov, Heinrich Heine University Dusseldorf, Germany. This work was supported by the Extreme Light Infrastructure Nuclear Physics (ELI-NP) Phase II, a project cofinanced by the Romanian Government and the European Union through the European Regional Development Fund - the Competitiveness Operational Programme (1/07.07.2016, COP, ID 1334).
[1] H. Habara, S. Honda, M. Katayama, H. Sakagami, K. Nagai, and K. A. Tanaka, Efficient energy absorption of intense pslaser pulse into nanowire target, Phys. Plasmas 23, 063105 (2016).

[2] S. Kahaly, S. K. Yadav, W. M. Wang, S. Sengupta, Z. M. Sheng, A. Das, P. K. Kaw, and G. R. Kumar, Near-Complete Absorption of Intense, Ultrashort Laser Light by Sub- $\lambda$ Gratings, Phys. Rev. Lett. 101, 145001 (2008).

[3] L. Cao, Y. Gu, Z. Zhao, L. Cao, W. Huang, W. Zhou, X. T. He, W. Yu, and M. Y. Yu, Enhanced absorption of intense shortpulse laser light by subwavelength nanolayered target, Phys. Plasmas 17, 043103 (2010).
[4] M. A. Purvis, V. N. Shlyaptsev, R. Hollinger, C. Bargsten, A. Pukhov, A. Prieto, Y. Wang, B. M. Luther, L. Yin, S. Wang, and J. J. Rocca, Relativistic plasma nanophotonics for ultrahigh energy density physics, Nat. Photonics 7, 796 (2013).

[5] C. Bargsten, R. Hollinger, M. G. Capeluto, V. Kaymak, A. Pukhov, S. Wang, A. Rockwood, Y. Wang, D. Keiss, R. Tommasini, R. London, J. Park, M. Busquet, M. Klapisch, V. N. Shlyaptsev, and J. J. Rocca, Energy penetration into arrays of aligned nanowires irradiated with relativistic intensities: Scaling to terabar pressures, Sci. Adv. 3, e1601558 (2017).

[6] J. H. Bin, M. Yeung, Z. Gong, H. Y. Wang, C. Kreuzer, M. L. Zhou, M. J. V. Streeter, P. S. Foster, S. Cousens, B. 
Dromey, J. Meyer-ter-Vehn, M. Zepf, and J. Schreiber, Enhanced Laser-Driven Ion Acceleration by Superponderomotive Electrons Generated from Near-Critical-Density Plasma, Phys. Rev. Lett. 120, 074801 (2018).

[7] N. Naumova, I. Sokolov, J. Nees, A. Maksimchuk, V. Yanovsky, and G. Mourou, Attosecond Electron Bunches, Phys. Rev. Lett. 93, 195003 (2004).

[8] Y.-Y. Ma, Z.-M. Sheng, Y.-T. Li, W.-W. Chang, X.-H. Yuan, M. Chen, H.-C. Wu, J. Zheng, and J. Zhang, Dense quasimonoenergetic attosecond electron bunches from laser interaction with wire and slice targets, Phys. Plasmas 13, 110702 (2006).

[9] G. Kulcsár, D. AlMawlawi, F. W. Budnik, P. R. Herman, M. Moskovits, L. Zhao, and R. S. Marjoribanks, Intense Picosecond X-Ray Pulses from Laser Plasmas by Use of Nanostructured "Velvet" Targets, Phys. Rev. Lett. 84, 5149 (2000).

[10] T. Nishikawa, H. Nakano, K. Oguri, N. Uesugi, M. Nakao, K. Nishio, and H. Masuda, Nanocylinder-array structure greatly increases the soft $\mathrm{x}$-ray intensity generated from femtosecondlaser-produced plasma, Appl. Phys. B 73, 185 (2001).

[11] A. Ovchinnikov, O. Kostenko, O. Chefonov, O. Rosmej, N. Andreev, M. Agranat, J. Duan, J. Liu, and V. Fortov, Characteristic $\mathrm{X}$-rays generation under the action of femtosecond laser pulses on nano-structured targets, Laser Part. Beams 29, 249 (2011).

[12] S. Mondal, I. Chakraborty, S. Ahmad, D. Carvalho, P. Singh, A. D. Lad, V. Narayanan, P. Ayyub, G. R. Kumar, J. Zheng, and Z. M. Sheng, Highly enhanced hard $\mathrm{x}$-ray emission from oriented metal nanorod arrays excited by intense femtosecond laser pulses, Phys. Rev. B 83, 035408 (2011).

[13] R. Hollinger, C. Bargsten, V. N. Shlyaptsev, V. Kaymak, A. Pukhov, M. G. Capeluto, S. Wang, A. Rockwood, Y. Wang, A. Townsend, A. Prieto, P. Stockton, A. Curtis, and J. J. Rocca, Efficient picosecond $\mathrm{x}$-ray pulse generation from plasmas in the radiation dominated regime, Optica 4, 1344 (2017).

[14] B. Martinez, E. d'Humières, and L. Gremillet, Synchrotron emission from nanowire array targets irradiated by ultraintense laser pulses, Plasma Phys. Controlled Fusion 60, 074009 (2018).

[15] V. Kaymak, A. Pukhov, V. N. Shlyaptsev, and J. J. Rocca, Nanoscale Ultradense $z$-Pinch Formation from LaserIrradiated Nanowire Arrays, Phys. Rev. Lett. 117, 035004 (2016).

[16] A. Curtis, C. Calvi, J. Tinsley, R. Hollinger, V. Kaymak, A. Pukhov, S. Wang, A. Rockwood, Y. Wang, V. N. Shlyaptsev, and J. J. Rocca, Micro-scale fusion in dense relativistic nanowire array plasmas, Nat. Commun. 9, 1077 (2018).

[17] B. Li, Z. Zhang, J. Wang, B. Zhang, Z. Zhao, L. Shan, W. Zhou, L. Cao, B. Zhang, and Y. Gu, Transport of fast electrons in a nanowire array with collisional effects included, Phys. Plasmas 22, 123118 (2015).

[18] F. Brunel, Not-So-Resonant, Resonant Absorption, Phys. Rev. Lett. 59, 52 (1987).

[19] C. Shukla and A. Das, Observation of enhanced absorption of laser radiation by nanostructured targets in pic simulations, Phys. Plasmas 24, 093118 (2017).
[20] M. Blanco, M. T. Flores-Arias, C. Ruiz, and M. Vranic, Tabletop laser-based proton acceleration in nanostructured targets, New J. Phys. 19, 033004 (2017).

[21] A. R. Bell, J. R. Davies, S. Guerin, and H. Ruhl, Fast-electron transport in high-intensity short-pulse laser - solid experiments, Plasma Phys. Controlled Fusion 39, 653 (1997).

[22] T. D. Arber, K. Bennett, C. S. Brady, A. Lawrence-Douglas, M. G. Ramsay, N. J. Sircombe, P. Gillies, R. G. Evans, H. Schmitz, A. R. Bell, and C. P. Ridgers, Contemporary particle-in-cell approach to laser-plasma modelling, Plasma Phys. Controlled Fusion 57, 113001 (2015).

[23] K. A. Tanaka, K. M. Spohr, D. L. Balabanski, S. Balascuta, L. Capponi, M. O. Cernaianu, M. Cuciuc, A. Cucoanes, I. Dancus, A. Dhal, B. Diaconescu, D. Doria, P. Ghenuche, D. G. Ghita, S. Kisyov, V. Nastasa, J. F. Ong, F. Rotaru, D. Sangwan, P.-A. Söderström et al., Current status and highlights of the eli-np research program, Matter Radiat. Extremes 5, 024402 (2020).

[24] W. L. Kruer and K. Estabrook, $\mathrm{J} \times \mathrm{b}$ heating by very intense laser light, Phys. Fluids 28, 430 (1985).

[25] See Supplemental Material at http://link.aps.org/supplemental/ 10.1103/PhysRevResearch.3.033262 for a video file of the simulation movie, comparison of the energy spectra, benchmark, and 3D simulation results.

[26] P. Gibbon, Short Pulse Laser Interactions With Matter: An Introduction (World Scientific, Singapore, 2005).

[27] A. Moreau, R. Hollinger, C. Calvi, S. Wang, Y. Wang, M. G. Capeluto, A. Rockwood, A. Curtis, S. Kasdorf, V. N. Shlyaptsev, V. Kaymak, A. Pukhov, and J. J. Rocca, Enhanced electron acceleration in aligned nanowire arrays irradiated at highly relativistic intensities, Plasma Phys. Controlled Fusion 62, 014013 (2019).

[28] E. Esarey, P. Sprangle, J. Krall, and A. Ting, Overview of plasma-based accelerator concepts, IEEE Trans. Plasma Sci. 24, 252 (1996).

[29] M. Sherlock, E. G. Hill, R. G. Evans, S. J. Rose, and W. Rozmus, In-Depth Plasma-Wave Heating of Dense Plasma Irradiated by Short Laser Pulses, Phys. Rev. Lett. 113, 255001 (2014).

[30] H. Burau, R. Widera, W. Hönig, G. Juckeland, A. Debus, T. Kluge, U. Schramm, T. E. Cowan, R. Sauerbrey, and M. Bussmann, Picongpu: A fully relativistic particle-in-cell code for a gpu cluster, IEEE Trans. Plasma Sci. 38, 2831 (2010).

[31] L. Di Lucchio and P. Gibbon, Relativistic attosecond electron bunch emission from few-cycle laser irradiated nanoscale droplets, Phys. Rev. ST Accel. Beams 18, 023402 (2015).

[32] L. D. Lucchio and P. Gibbon, Post-acceleration of electron bunches from laser-irradiated nanoclusters, Phys. Scr. 96, 055603 (2021).

[33] M. T. Li, G. Y. Hu, L. G. Huang, and J. Zheng, Simulation of hard $\mathrm{x}$-rays source produced by a picosecond laser irradiated solid target for compton radiography, Plasma Phys. Controlled Fusion 62, 095006 (2020).

[34] X. Zhang, T. Tajima, D. Farinella, Y. Shin, G. Mourou, J. Wheeler, P. Taborek, P. Chen, F. Dollar, and B. Shen, Particlein-cell simulation of $\mathrm{X}$-ray wakefield acceleration and betatron radiation in nanotubes, Phys. Rev. Accel. Beams 19, 101004 (2016). 